\section{$\underset{\substack{\text { hommes } \\ \text { \& migrations }}}{ }$}

\section{Hommes \& migrations}

Revue française de référence sur les dynamiques

migratoires

$1309 \mid 2015$

Le $3 e$ âge des migrants

\title{
Respect du corps et des souhaits de sépulture des Maghrébins âgés
}

\section{Anne-Marie Duguet et Jenny Duchier}

\section{(apenEdition \\ Journals}

\section{Édition électronique}

URL : http://journals.openedition.org/hommesmigrations/3097

DOI : 10.4000/hommesmigrations.3097

ISSN : 2262-3353

\section{Éditeur}

Musée national de l'histoire de l'immigration

\section{Édition imprimée}

Date de publication : 1 janvier 2015

Pagination : 147-154

ISBN : 978-2-919040-30-8

ISSN : $1142-852 X$

\section{Référence électronique}

Anne-Marie Duguet et Jenny Duchier, « Respect du corps et des souhaits de sépulture des Maghrébins âgés », Hommes \& migrations [En ligne], 1309 | 2015, mis en ligne le 01 janvier 2017, consulté le 19 avril 2019. URL : http://journals.openedition.org/hommesmigrations/3097 ; DOI : 10.4000/hommesmigrations.3097 


\title{
RESPECT DU CORPS ET DES SOUHAITS DE SÉPULTURE DES MAGHRÉBINS ÂGÉS
}

par ANNE-MARIE DUGUET, maître de conférences des universités, et Jenny Duchier, ethnologue ingénieur d'études (UMR 1027 Inserm, université Paul-Sabatier, Toulouse).

\author{
Les familles des migrants musulmans âgés, en particulier ceux \\ atteints de troubles cognitifs, demeurent réticents à placer leurs \\ aînés en milieu hospitalier. En cause, la crainte qu'on ne tienne \\ pas compte de leur culture quant au respect du corps et aux rites \\ funéraires. Les personnels soignants commencent à prendre \\ conscience de leur manque de connaissances des us et coutumes \\ musulmans afin de prévenir ce type de discriminations.
}

Les professionnels de santé, questionnés pour l'étude MATC ${ }^{1}$ au sujet de l'incidence de la culture sur les soins aux patients âgés originaires du Maghreb, ont souligné qu'ils étaient soucieux de respecter au mieux la culture et la religion de ces patients, mais aussi que, face à une pathologie souvent trop avancée pour qu'ils expriment eux-mêmes des souhaits, ils ignoraient les attentes réelles de leurs familles. En dehors des professionnels originaires de ces pays, les connaissances des autres membres du personnel se limitent aux restrictions dans l'alimentation (pas de porc, ni d'alcool) et aux rites funéraires (pas d'atteinte à l'intégrité du corps, toilette rituelle). Tous les professionnels constatent que les Maghrébins sont très peu nombreux dans les structures d'accueil telles que les établissements d'hébergement pour personnes âgées dépendantes (EHPAD) et les accueils de jour, et qu'il y a une "discrimination" de fait, en partie parce que, craignant que leurs aînés ne trouvent pas de repères sécurisants dans des structures inadaptées, les familles préfèrent les garder au domicile, respectant ainsi leur devoir de piété filiale traditionnelle. De son côté, le personnel, ne recevant que très rarement ces patients, n’a pas l'expertise nécessaire et déplore le manque de formation à cet égard.

Toutefois, dans les services d'urgences des hôpitaux, les Maghrébins âgés constituent une part non 
négligeable des admissions (autour de 20\%, selon les établissements) quand leur état de santé s'aggrave brutalement. Dans ces circonstances, l'accompagnement de fin de vie et du décès soulève des questionnements que nous tenterons d'analyser.

\section{Entretiens de l'étude MATC: culture et soins aux migrants âgés}

Létude MATC, Incidence des facteurs socioculturels sur les besoins et la prise en charge des migrants âgés originaires d'Afrique du Nord atteints de troubles cognitifs (maladie d'Alzheimer et maladies apparentées), soutenue par la Fondation Médéric Alzheimer, a pour objet d'identifier les freins à l'entrée des migrants âgés originaires d'Afrique du Nord dans les dispositifs

En principe, c'est l'épouse qui s'occupe de la toilette de son mari. Le personnel soignant accepte, pour ne pas heurter la personne âgée, que l'épouse se rende à l'hôpital pour effectuer personnellement la toilette. gérontologiques d'aide et de soins et les EHPAD, et d'évaluer la part des inégalités sociales de santé et celle des traditions et de la culture.

Il s'agit d'une étude socioanthropologique auprès de familles, de services hospitaliers - l'Unité d'hospitalisation de courte durée (UHCD) de Toulouse, l'Unité de soins de longue durée (USLD) et le Centre de soins de suite et de rééducation (SSR) de Draveil -, et de services médicosociaux. Parallèlement, une enquête par questionnaires a été réalisée à l'hôpital de Sfax (Tunisie) auprès des professionnels en charge des patients souffrant de ces pathologies. Cette étude est complétée par un recueil d'expériences par entretiens de médecins et de professionnels en région parisienne, à Toulouse et à Lavelanet (Ariège).

L'objectif de l'étude MATC n'était pas de questionner directement les souhaits de sépulture. Cependant, au détour des entretiens sur les questions relatives à la culture, des éléments pertinents pour notre réflexion sur le respect du corps, le décès et les rites funéraires ont été évoqués. Cette étude est qualitative dans le but d'identifier le ressenti des patients, des familles et des professionnels.

Pour les migrants âgés et leur entourage, le rôle de la famille est très important, le respect des anciens est toujours présent et les traditions doivent être prises en compte pour que les patients ne soient pas heurtés. Certains professionnels des institutions ignorent la culture et les traditions du Maghreb et craignent de ne pas avoir l'attitude attendue par manque d'expérience. Toutefois, la présence parmi les professionnels interrogés de personnes originaires d'Afrique du Nord a permis de nuancer ces propos et de souligner l'influence des représentations sociales sur les migrants âgés dans les institutions et les inégalités induites.

\section{Respect du corps dans la culture du Maghreb}

Établir une bonne relation avec les sujets âgés et les familles semble passer par une connaissance et un respect des dimensions culturelles. Pour les soins, les personnels de la même culture sont en général mieux acceptés. "Pour les personnes âgées venant du Maghreb, le respect de la pudeur et la protection de la nudité sont primordiaux. Le regard d'une femme, surtout étrangère, sur le corps d'un homme est un sacrilège." L'infirmière libérale qui tient ces propos nous déclare qu'il lui est arrivé de donner des douches à un homme maghrébin malade qui gardait ses vêtements... Elle ajoute : "Pendant les soins infirmiers à domicile, toute la famille se retire." Certaines familles demandent que les soins infirmiers soient interrompus pendant le ramadan.

Un urgentiste d'origine musulmane nous a signalé des cas dramatiques liés au manque d'hygiène (macération, escarres, infections) des hommes maghrébins âgés admis pour une pathologie aiguë, parce qu'aucun membre de la famille n'osait le dénuder pour la toilette intime, ou bien parce qu'ils 
refusaient que l'on s'approche d'eux dans ce but. En principe, c'est l'épouse qui s'occupe de la toilette de son mari. Le personnel soignant accepte, pour ne pas heurter la personne âgée, que l'épouse se rende à l'hôpital pour effectuer personnellement la toilette.

Les soins à la femme âgée sont plus facilement confiés à un professionnel extérieur, même si c'est habituellement la fille ou la belle-fille qui s'en charge. Quand une aide à domicile est acceptée, il s'agit le plus souvent d'une femme d'âge mûr.

Pour les patients atteints de troubles cognitifs, les professionnels interrogés en Tunisie (17 médecins et 1 psychologue) ont signalé que les familles plaçaient en premier les questions d'hygiène du corps (pour 11 professionnels) pour justifier une aide, au même titre que les traitements médicaux adaptés. Une attention particulière devrait être portée aux conditions de réalisation des soins corporels, même si le respect de la personne doit être identique quelle que soit la culture. L'impact psychologique des maladresses par méconnaissance pourrait rendre pénible le séjour en institution.

\section{L'accompagnement dans la fin de vie}

La fin de vie est entièrement gérée par la famille, qui reste auprès de la personne et l'entoure en respectant les traditions. Toutefois, une infirmière libérale dit que ces habitudes se rencontrent surtout à l'égard des personnes les plus âgées, pour lesquelles la culture familiale traditionnelle prédomine, et qu'elles sont en train de changer avec les nouvelles générations.

Un Algérien de 35 ans, né en France, a assisté à la fin de vie de sa grand-mère centenaire en Algérie. Toute la famille était présente et s'est relayée auprès d'elle jusqu'au dernier soupir. Il n'était pas d'accord avec le fait qu'on ait gardé sa grand-mère à domicile. Les traditions familiales qui perduraient en Algérie étaient, à ses yeux, excessives. Il aurait préféré éviter des souffrances à sa grand-mère en l'hospitalisant, mais il reconnaît qu'il ne savait pas vraiment les soins qu'elle aurait pu y recevoir. Ce témoignage illustre bien la situation de transition avec, d'un côté, pour les plus jeunes, le désir de limiter les souffrances de fin de vie par des soins médicaux et, de l'autre, le respect des usages qui organisent la présence des proches pour accompagner le mourant.

Si un patient n'a plus de famille, les professionnels maghrébins de notre étude qui travaillent dans des services gériatriques en région parisienne ne se sentent pas autorisés à l'assister d'un point de vue religieux, même s'ils connaissent les rites, parce qu'ils considèrent que ce serait La fin de vie est entièrement gérée par la famille, qui reste auprès de la personne et l'entoure en respectant les traditions. contraire au principe de laïcité de l'hôpital. On peut le regretter parce que, comme le dit Atmane Aggoun², mourir seul à l'hôpital est angoissant, même s'il y a une infirmière, parce qu'elle ne sait (ou ne peut) pas réciter la première sourate du Coran, essentielle au moment du dernier souffle. Pour un musulman, tenir la main ne suffit pas, il faut aussi maintenir l'index droit levé (geste qui signifie la croyance en l'unicité de Dieu). Les mêmes professionnels révèlent le manque de repères culturels (pièce de prière, lien avec l'imam local, fond de musique coranique...) qui permettraient d'accueillir le patient dans un milieu apaisant pour ses derniers jours. Le principe de laïcité n'exclut pas le soutien religieux pendant l'hospitalisation, c'est un droit du patient. Au centre hospitalier universitaire (CHU) de Toulouse, pour les patients de confession musulmane, un calendrier détaillé des horaires de prières est fourni, avec les dates des fêtes religieuses et du ramadan. Le matériel nécessaire à l'accomplissement de la prière est mis à disposition (tapis de prière, indication de la direction de la Qibla pour la prière...), mais rarement demandé. Le mufti peut être contacté pour des questions d'ordre religieux susceptibles d'être posées dans l'aide à la décision pour les soins. Il 
n'est pas certain que les familles soient informées de ces dispositions. En réalité, certaines familles $^{3}$ considèrent les institutions d'hébergement médico-sociales comme incompatibles voire hostiles à leur conception de l'accompagnement de leurs parents lors de leurs derniers jours.

\section{Nourriture, rites funéraires : le respect des traditions et de la culture musulmanes}

La question de l'alimentation fournie par les établissements d'hébergement ou de soins est souvent citée comme une préoccupation des familles. Un gériatre précise que, dans son service, les familles sont autorisées à apporter de la nourriture halal. Un cuisinier se dit respectueux des impératifs et fait en sorte de se conformer aux critères de séparation dans la préparation des repas et de fournir des produits halal, en accord avec

$\mathrm{Au} \mathrm{CHU}$ quand un patient musulman décède, l'aumônier

peut assister la famille du défunt lors des ablutions mortuaires et de la prière selon le rite musulman.

famille. Une surveillante responsable des chambres mortuaires du CHU de Toulouse nous a précisé que tous les patients musulmans qui décèdent bénéficient d'une toilette rituelle quel que soit leur âge y compris les fœetus. Environ tous les deux jours, une toilette rituelle est organisée par les familles dans une chambre dédiée, avec l'intervention de services spécialisés de pompes funèbres ou avec l'imam du CHU. La sortie du corps après la mort se fait dans un délai de 24 à 48 heures. Cela n'est pas en conformité avec la tradition musulmane, qui requiert une inhumation très précoce (contraire à la législation funéraire en France). Les trois quarts des personnes décédées au CHU sont rapatriées dans leur pays de naissance. Un quart seulement sont inhumées en France. Les soins de conservation ne sont pas compatibles avec le rite musulman, mais, s'ils sont requis, ils sont effectués a minima pour le transport international.

À l'opposé, le personnel des services de soins n'a pas de connaissances précises sur les dispositions à prendre pour le décès des personnes de confession musulmane. Les familles se trouvent isolées au moment du décès tant qu'elles n'ont pas rencontré des professionnels spécialisés. Les spécialistes du funéraire musulman ont un accueil 24 heures sur 24. Les familles ont souvent anticipé le décès de longue date en se cotisant pour le rapatriement du corps et ont déjà prévu l'organisation de la toilette.

$\mathrm{Au}$ CHU quand un patient musulman décède, l'aumônier peut assister la famille du défunt lors des ablutions mortuaires et de la prière selon le rite musulman. Une équipe spécialisée sous la tutelle du mufti officiel du Sud-Ouest peut l'accompagner dans les étapes techniques et administratives.

\section{Droits des patients et déontologie}

Les différents codes de déontologie des professions de santé érigent en devoir fondamental le respect de la dignité de la personne, au même titre que le respect de la vie.

Pour les médecins, l'article 2 dit : "Le médecin, au service de l'individu et de la santé publique, exerce sa mission dans le respect de la vie humaine, de la personne et de sa dignité. Le respect dû à la personne ne cesse pas de s'imposer après la mort" (R-4127-2 CSP).

La dignité est considérée comme intrinsèque à tout être humain qui doit être soigné avec la même conscience, sans distinction selon l'état de santé ou le handicap, et sans discrimination sur son origine, ses mœurs, sa situation de famille, son appartenance ou non à une ethnie, une nation, une 
Layachi Ait-Baaziz dans son nouvel appartement de $30 \mathrm{~m}^{2}$ situé dans le XIII arrondissement de Paris, 30 mars 2015 @ Camille Millerand

religion (art. R 4127-7 CSP). En fin de vie, le médecin doit accompagner le mourant, assurer la qualité d'une vie qui prend fin et sauvegarder la dignité (art. R 4127-38 CSP). Les infirmiers ont les mêmes devoirs que les médecins, ils agissent dans le cadre de leur rôle propre et ont toute latitude pour fournir des soins respectueux (art. R 4311-3 CSP). La loi du 4 mars 2002 sur les droits des malades a repris ces principaux devoirs en les transposant en droits pour les patients : le droit au respect de la dignité (art. L 1110-2), à la non-discrimination (L 1110-3) et au respect de la vie privée (L 1110-4). La culture et la religion sont du domaine de la vie privée. La chambre du patient à l'hôpital est un lieu privé dans lequel il peut accomplir ses traditions. Toutefois, les impératifs liés aux soins restreignent très largement le champ des libertés culturelles. Il est parfois difficile d'organiser le soutien spirituel, quelle que soit la religion.

Respecter la dignité du patient, c'est le traiter d'égal à égal et ne pas le placer en situation d'infériorité. Les soignants doivent protéger tous les intérêts de la personne, son intégrité corporelle, psychologique et culturelle. Le patient hospitalisé est vulnérable en raison des contraintes liées à la grande technicité des soins et au fonctionnement de l'institution qui l'accueille. Le sujet est 
particulièrement fragilisé dans les situations de douleur intense, d'angoisse majeure, de confusion mentale, de perte de conscience (ou d'anesthésie) et de coma. Le patient n'est plus maître de lui et de ses choix, ses aptitudes sont amoindries. Le respect de la dignité d'un sujet sans défense dépend de la bonne volonté des soignants qui doivent faire à la place du sujet les gestes de la vie courante, de la toilette, des soins du corps, dans le respect des souhaits du malade. Le recours à la famille pour connaître les volontés de la personne est primordial quand celle-ci ne peut pas s'exprimer.

\section{Le corps après la mort : concilier les convictions}

Dans la littérature sur les traditions de l'islam relatives au respect du corps et à la mort, nous ne retiendrons ici que les éléments qui éclairent les questionnements soulevés lors des entretiens.

La propreté, par exemple, fait partie de la foi. Le musulman se purifie cinq fois par jour au moment de la prière. La propreté suit l'être humain tout au long de sa vie, de la naissance à la mort, et les proches assurent la der-
La crainte de l'exhumation avant que tous les os ne soient

devenus poussière est une

des justifications

de l'inhumation au pays, car on est sûr que le corps enterré ne sera pas déplacé nière toilette avant l'ensevelissement. Lors du déshabillage pendant le rite funéraire ${ }^{4}$, les parties intimes doivent être couvertes par un linge pour l'homme du nombril à la moitié de la cuisse et pour la femme de la poitrine au genou. La toilette est effectuée avec un gant de toilette. Ce rite traduit l'importance accordée au respect du corps et signe l'absence de contact direct avec les parties intimes, qui pourrait expliquer les précautions extrêmes voire l'incapacité pour l'entourage de prodiguer des soins d'hygiène à l'homme âgé ou à la femme âgée. Ce respect de l'intimité va au-delà de la pudeur, certaines personnes âgées lui attribuent un fondement religieux, puisque la nudité ne s'expose pas, et les nécessités des soins dans certaines circonstances peuvent les heurter. Selon Atmane Aggoun, le choix crucial pour un migrant est celui du lieu de son inhumation. "Être enterré au village c'est sacraliser la terre ${ }^{5}$." Les parents rapatrient même les corps des jeunes qui ne sont jamais allés au village pour leur faire "une belle mort" après leur décès en France d'accident de voiture, d'overdose ou de sida. Le déplacement du corps après la mort est proscrit par l'islam puisqu'on devrait être enterré à l'endroit où on est mort. Le corps enterré ne doit plus être déplacé. Il faut donc disposer de concessions à perpétuité pour assurer le repos de l'âme du mort. La crainte de l'exhumation avant que tous les os ne soient devenus poussière est une des justifications de l'inhumation au pays, car on est sûr que le corps enterré ne sera pas déplacé. Si, toutefois, il s'avérait indispensable d'exhumer, il conviendrait de les regrouper de manière confessionnelle dans des ossuaires musulmans. Ce respect de l'entourage confessionnel des restes humains a été consacré par la décision du tribunal de grande instance (TGI) de Lille reconnaissant que "la dépouille mortelle demeure un objet de respect dont le caractère sacré est rappelé par l'article 16-1 du Code civil : la loi assure la primauté de la personne et interdit toute atteinte à la dignité de celle-ci (...). La paix due aux morts n'est pas respectée si, lors d'une exhumation, par erreur les restes d'une personne de confession musulmane sont mis dans une fosse commune avec ceux de personnes d'autres confessions $^{6 "}$. Le 4 juillet 1990, les services de la mairie avaient retiré la dépouille de Madame $\mathrm{K}$. de la concession de son gendre, qui avait été renouvelée jusqu'en 2005. Ils avaient mis une autre sépulture à la place. Une indemnisation de 1500 euros a été versée pour préjudice moral à la veuve du titulaire de la concession et 500 euros à chacun des petitsenfants. 1000 euros ont aussi été attribués au titre de l'atteinte à la dignité de la dépouille mortelle. 


\section{Les lieux d'inhumation des musulmans en France}

La question des cimetières confessionnels seposeen France. Iln'est pas permis aux maires d'établir des distinctions ou des prescriptions particulières en raison des croyances ou du culte du défunt ou des circonstances qui ont accompagné sa mort ${ }^{7 ”}$.Le maire ne peut autoriser "linhumation d'un défunt dans un espace donné du cimetière communal, au seul motif de son appartenance religieuse, ce qui paraît contraire au principe constitutionnel d'égalitê". Cependant, au titre de la liberté de religion, le défunt devrait pouvoir être enterré selon ses choix. Le cimetière franco-musulman de Bobigny ${ }^{8}$ est le seul cimetière entièrement consacré à des sépultures musulmanes en France. Il a été créé en 1936, faisant suite à l'ouverture de la Mosquée de Paris en 1926 et à celle de l'hôpital franco-musulman de Bobigny en 1935. Le cimetière est une annexe privée de l'hôpital. Un décret de 1937 autorise le recteur de la Mosquée de Paris à demander des inhumations. En 1962, le cimetière passe sous la tutelle de l'Assistance publique et tombe dans l'abandon dans les années 1970. Réhabilité en 1996, il est devenu un carré musulman du cimetière intercommunal.

Le cimetière de Thiais (Val-de-Marne) dispose d'un carré musulman très important. La seule différence qui caractérise le carré par rapport au reste du cimetière, c'est l'orientation des tombes vers La Mecque et les concessions perpétuelles.

Il y aurait, en France, 300 "espaces confessionnels" dans les cimetières ${ }^{9}$, dont 200 "carrés musulmans" et une centaine de "carrés israélites". Le ministère de l'Intérieur estime que le nombre d'"espaces confessionnels" a connu une réelle augmentation au cours des dernières années.

Messaoud Khlalo (à gauche), un Algérien de 87 ans,

au foyer Adoma “Les Grésillons", à Gennevilliers, 1 ${ }^{\text {er }}$ avril 2015.

(c) Camille Millerand

\section{La réponse des institutions}

Les pouvoirs publics se sont saisis de ces questions. En premier lieu, dans son rapport de 2010, le défenseur des droits s'est intéressé à l'institution d'espaces confessionnels au sein des cimetières municipaux et s'est inquiété des discriminations qui ont pu survenir. Alexis Bachelay a été chargé de diriger un groupe de réflexion parlementaire en 2013.

Le défenseur précise que les regroupements confessionnels "de fait" ont été encouragés par des circulaires, même si cela ne correspond pas au droit. Le médiateur de la République a été saisi en $2009^{10}$ d'une réclamation émanant d'une personne de confession musulmane qui souhaitait acquérir une concession au sein du carré musulman du cimetière municipal. La commune n'a pas accédé à sa demande, puisqu'il n'y avait pas d'espace confessionnel. 
Aucun dysfonctionnement administratif n'a pu être établi, parce que le maire a toute liberté en matière de délivrance des concessions funéraires. Le médiateur a invité la commune à informer le réclamant qu'un carré musulman existait dans une commune voisine afin qu'il y présente sa demande à titre dérogatoire.

Selon le rapport Bachelay, le choix du lieu de sépulture crée un conflit entre le respect de la tradition et l'application de la loi.

Le rapport souligne que, de plus en plus, les Maghrébins veulent être inhumés en France, mais l'inhumation selon les rites religieux dans des carrés confessionnels engendre des problèmes juridiques, et il n'existe aucune certitude de pouvoir être inhumé selon ses volontés.

\section{Les difficultés juridiques}

Les communes qui disposent de carrés confessionnels se trouvent parfois dans l'incapacité de répondre favorablement aux nombreuses demandes d'inhumation émanant notamment des résidents de communes voisines ${ }^{11}$. Il semble que "le regroupement de fait de sépultures, comme décision individuelle", ne soit pas prohibé par la loi, ce qui permet de pérenniser des situations existantes et, selon le souhait du rapporteur Bachelay, d'améliorer la situation en créant des regroupements confessionnels de fait en nombre suffisant après avoir évalué les besoins.

Les familles sont contraintes de choisir "entre le renvoi du corps dans le pays d'origine, considéré comme trop onéreux par certaines d'entre elles, et l'inhumation du défunt en France, sachant que les règles propres au culte (orientation des tombes, durée illimitée des sépultures, etc.) peuvent ne pas être satisfaites ${ }^{12 "}$.

L'enterrement en France a été validé par les autorités religieuses musulmanes et fait désormais partie du projet de vie de certains immigrés. En outre, l'inhumation en France -particulièrement celle des femmes - est d'autant plus fréquente que les enfants, souvent de nationalité française, y résident.

Par ailleurs, reprenant l'analyse de Chaïb Yassine, Alexis Bachelay mentionne que les pouvoirs publics doivent rendre possible l'"enracinement" de la première génération d'immigrés, sinon la question du choix du lieu d'inhumation se posera immanquablement à la deuxième génération, pourtant née en France. De plus, la mise en place d'“espaces confessionnels" en nombre suffisant serait susceptible d'apaiser les craintes liées à la mort en terre d'accueil parfois ressenties par les immigrés vieillissants.

\section{Conclusion}

Il ressort de notre étude qu'une véritable demande existe chez les professionnels pour une sensibilisation aux besoins des personnes originaires du Maghreb, qui devrait porter sur la culture et la relation aux soins, avec une mention spéciale pour l'attitude à avoir en fin de vie et pour le soutien à apporter aux familles dans leur deuil en leur donnant des informations pertinentes.

La question de la dignité du corps et du respect des souhaits pour l'inhumation se pose pour toute personne, quelles que soient sa religion, sa spiritualité et sa culture. C'est la signification de la vie sur terre, de la mort et du respect des personnes qui est en jeu. D'une façon récurrente, les acteurs médico-sociaux associent les problèmes d'accès aux soins à des "différences de traitement", à une certaine "réticence inconsciente" sur la situation des vieux issus de l'immigration. Une "compétence culturelle" favorisant les interventions des professionnels et une adaptation environnementale à des considérations culturelles spécifiques paraissent nécessaires dans les institutions pour faire bénéficier les migrants âgés atteints de la maladie d'Alzheimer ou d'une maladie apparentée des prestations auxquelles ils ont droit dans le respect de leurs codes culturels. 